\title{
Correlative Tomography - Extraction of Reliable Information with Adequate Resolution from mm Scale Down to Sub-nm Scale
}

\author{
Frank Mücklich $^{1,2}$, Johannes Webel ${ }^{1}$, Hisham Aboulfadl ${ }^{1}$, N. Lindow ${ }^{3}$, H.-C. Hege ${ }^{3}$ \\ 1. Department Materials Science and Engineering, Saarland University, Saarbrücken, Germany \\ 2. Material Engineering Center Saarland (MECS), Steinbeis Research Center, Saarbrücken, Germany \\ 3. Department of visualization and data analyses, Zuse Institute Berlin, Berlin, Germany
}

Most of the basic quantitative parameters of the 3D microstructure such as volume ratio of different phases, specific interfaces or mean curvatures, could be estimated by applying stochastic geometry and stereology on planar sections using well known metallographic techniques in various scales. If the geometry of the microstructure and the topology are very complex, the tomographical approaches are indispensable, since they open access to image the 3D shape and arrangement of microstructural objects, as well as estimating the total curvature [1]. In order to understand how the underlying microstructure is formed during the processing and how it determines the very specific set of properties of today's highperformance materials, it is essential to analyze and quantify all the key features of the microstructures in three dimensions in a relevant scale using correlative tomography.

For correlative microscopy, a lot of techniques are available which are mainly based on light microscopy, electron microscopy and scanning probe microscopy. Each of the analytical techniques used for correlative microscopy differ not only in the resolution and information content, but also significantly vary in the sampling volume. The non-destructive methods such as X-ray tomography (Lab-CT/Synchrotron CT) and electron tomography typically have a trade-off for local resolution versus the sampling volume. While electron tomography and atom probe tomography (APT) have a trade-off between the structural and chemical sensitivity verses the sampling volume [2].

It turns out that the serial section techniques, such as FIB-SEM tomography and metallographic serial sectioning tomography offer powerful tools to bridge the critical gaps between other tomographic techniques. They have a unique potential to combine their typical resolution with a relatively huge sampling volume (up to $\sim 10^{8} \mu \mathrm{m}^{3}$ ). The paper will discuss the potential and the limits of a fully reproducible high resolution metallographic serial sectioning tomography, which opens the possibility to investigate large volumes and help reveal unexpected correlations of microstructure formation [3].

Apart from the sampling volume, which is around $10^{-4} \mu \mathrm{m}^{3}$ for an APT specimen, an improved understanding from the correlation of such small volume with data from higher scale levels requires extracting as much information as possible from the measured APT data. The limited detection efficiency added to the limited capability of rendering complete datasets increases the need for using new visualization solutions, which would help revealing nano-segregations and complex morphologies in the datasets. Better identification of microstructural features at the sub-nanoscale, such as lattice defects or complex interfaces, result in an improved understanding of the micro- to macro- structural correlations. We present here some new techniques for rendering large APT datasets (tens of millions of sphere-shaped atoms) at high interactive speeds [4], as well as Fourier transformation algorithms for identifying lattice defects in the reconstructions. 
[1] J Ohser and F Mücklich, Statistical Analysis of Microstructures in Materials Science, John Wiley \& Sons, Chichester, (2000).

[2] L. Arslan, et al, Ultramicroscopy 108 (2008) 1579-1585.

[3] J Webel, Development of a Light Microscopy Serial Sectioning Tomography (in German), Bachelor Thesis, Saarland University, (2014).

[4] N. Lindow, D. Baum, H.-C. Hege, Eurographics Conference on Visualization 31 (2012) 1325-1334

[5] The authors acknowledge the funding from the German science foundation, MU 959/29.

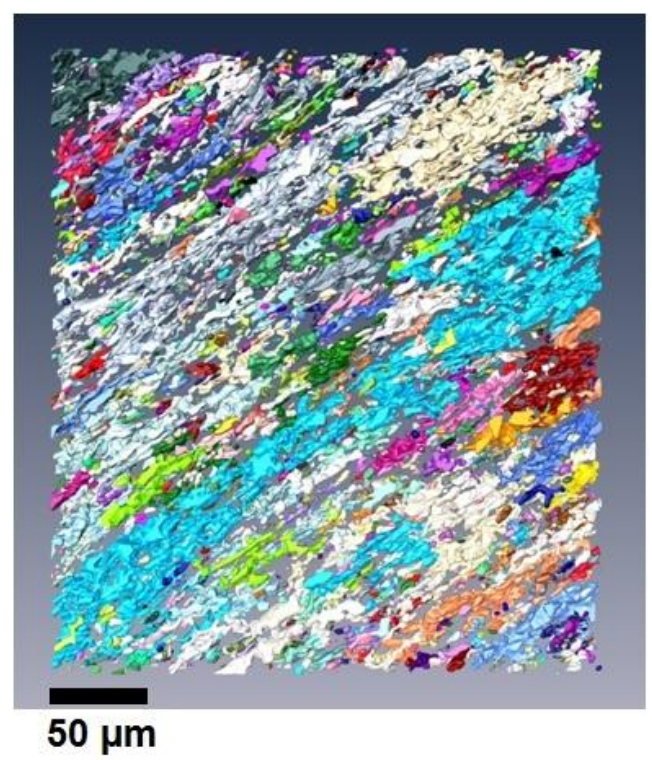

Figure 1. A reconstruction for metallographic serial sectioning tomography data from a steel sample. Each color represents a specific interconnected particle in the sample.

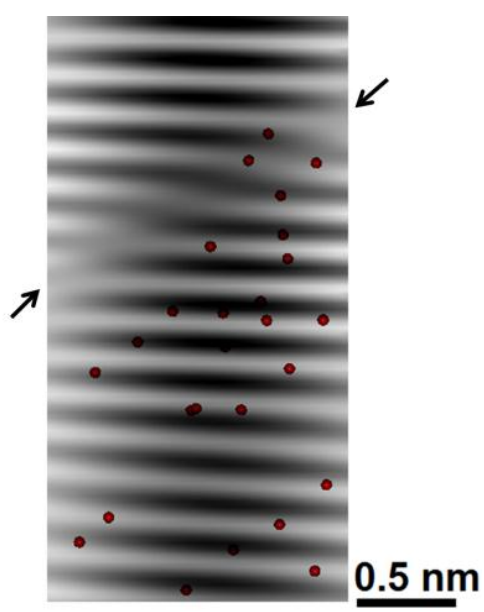

Figure 2. Filtered localized region from an APT dataset after Fourier transformation, highlights lattice planes from a crystallographic pole along (100) direction in an Al alloy. A disturbed region (marked with arrows) represents an edge dislocation. Red spheres are solute atom diffusion to the dislocation strain energy. 